\title{
DEMOCRACY AND TRUTH: A CLUSTER OF PROBLEMS - AN ATTEMPT TO SORT THEM OUT ${ }^{1}$
}

\begin{abstract}
This paper attempts to show that an idea of truth and a normative liberal democratic theory can go hand in hand. First, it is argued that truth has something to do with politics. Second, three possible aspects of the relationship between democratic government and truth are discussed. The discussion begins with a presentation of arguments for the view that the process of establishing scientific truth stricto sensu is not generally a matter of wide public or democratic debate, and thus is not incompatible with a democratic order. The discussion then moves on to consider practical social problems; it endswith the claim that unlike in "pure" scientific inquiry, democratic procedure is directly relevant and perhaps crucial to solving practical collective problems. The final part of the paper concerns "the market of ideas" and the possibility of its failure. It is clear that market of ideas is not sufficient for producing autonomous and critical citizens who have a ,strong desire not to be fooled" (Bernard Williams). Quite the contrary, the marketplace of ideas may be exposed to agencies that deliberately distort and conceal the truth. Governments are prone to commit such illegitimate actions because of the very nature of their ways of working. This may be the most difficult problem for a democratic institutional framework.
\end{abstract}

Keywords: truth, politics, democracy

\section{Introduction}

Perhaps we should begin our discussion with the alleged dangers of truth for democracy. For example, many important political philosophers (Hannah Arendt, Karl Popper, and John Rawls, to name but a few) worried about the potential authoritarian and totalitarian implications of the concept of truth in politics. On this view, truth has a coercive nature that is unfriendly to the spirit of tolerance necessary for democratic political life that acknowledges valuepluralism in a society. In a similar vein but much earlier, John Locke - in his Letter Concerning Toleration - expressed the view that the function of law is not

1 Work on this paper was supported by the project "Dynamic Systems in Nature and Society: Philosophical and Empirical Aspects" (No. 179041), financed by Ministry of Education, Science and Technological Development of the Republic of Serbia. 
to secure the truth of citizens' opinions but to provide stable and longlasting government where believers of various kinds can simultaneously practice their religious commitments.

Suppose that for certain reasons we want to keep the term 'truth' in a theoretical debate about the nature of the political. Offhand, it seems that politics has something to do with truth. An argument can be developed in the following way: assuming that life's being worth living has something to do with the conditions of the world we inhabit, which may be so because most of us want to cultivate the world around us by cooperating with others in light of, say, visions of the good life, it seems that we should be concerned with having adequate information about the world, that is to say, we cannot avoid truth claims, or, at least, some probabilistic estimates about the way the world is. In addition, we should see to it that the structure of our collective endeavour to attain a good life fits the demands of the task. In this sense we can say that a political structure is bad or good, and that the outcome of the working of its institutions are successes or failures. And this may a way to pull truth between Scylla and Charybdis of the abovementioned liberal worries. Perhaps the answer is to rely on minimal, commonsensical, and pragmatic conceptions of truth. Instead of using the term 'truth' in a "heavy realist" manner, we can start out with the idea of hypothetical objectivity (or, with an ,independent standard of correctness") ${ }^{2}$ in order to make sense of what we do in science and politics. Both scientific inquiry and politics are understood as problem-solving activities; „standards of correctness” in their various forms and character pertain to these activities. The question is how democratic society and requests for truth understood in this "light” sense interlock. That is to say, we then have to ask how truth comes into play into politics, and what democratic politics needs to do such that truth comes into politics in the right way.

When answering this question, there are several aspects of the problem that need to be clarified. I'll name them one by one as they come naturally, without false hope of exhausting the list. Let us first begin by delineating the aspects of the problem. Three aspects will be discussed here: 1) the relationship between scientific inquiry and democratic society; 2) solving practical social problems; and 3 ) the problem of creating autonomous citizens (the relationship between the "marketplace of ideas” and democratic political culture).

\section{Independent Objectivity as a Target: From Scientific Theories to Moral Principles}

It seems that the way we identify the aspects of the relationship between truth and democracy depends on how we see the character of truth claims. There is a huge variety of issues with which politics is concerned. Issues that demand political action could be classified in a series of items that starts out with, say, deciding on where and how to build bridges, and then goes over

2 Recent work by Helene Landemore relies on this idea. See her Democratic Reason: Politics, Collective Intelligence and the Rule of the Many, Princeton, Princeton University Press, 2013. 
problems such as global warming, putting a drug on the market, the choice of tax-system, going to war, immigration, and then finishes, say, with deciding on allowing euthanasia in hospitals, same-sex marriage, capital punishment, and so on. On the one side of this imagined spectrum there are deep moral, religious and political disagreements and a diversity of citizens' opinions on how to solve them, and, on the other, there are opinions and judgements that are more about natural facts and other scientific claims. ${ }^{3}$ The imagined spectrum can be extended by adding scientific inquiry that addresses issues with almost no political bearing. For example, we can think of a scientist who works on the discovery of subatomic particles or on structure of DNA who is motivated solely by theoretical questions about the structure of matter, or, say, the beginning of life. If this is so, we can define our spectrum along the line that begins from basic or "pure” scientific inquiry, which has only epistemic significance, and which continues with examples of applied science with both epistemic and practical significance, and ends up with activity that addresses practical social problems that have deep moral significance.

In light of all this, we can generate two claims: first, that the range and variety of issues corresponds to the domain with which they are associated; second, that the method of solving the issues, when done correctly, is closely knit with the nature of the domain-i.e., it is hard to imagine a non-trivial, general standard across the board.

There is one more point that needs to be made clear. Even if we suppose that there is no unqualified and stateable truth across the board, taking the form of a "substantial something" out there in the world that makes all our judgments true or false, we have to concede that the status of the judgments about the issues (good/bad judgements; correct/incorrect) depends on how far they are from something we might hypothetically call „objectivity”. When we speak of natural science, this term could refer to scientific facts and laws that are the subject matter of the entire explanatory framework ${ }^{4}$ within a certain field; but it can also refer to the normative claims that themselves yield practical objectivity. These latter normative claims can be understood as putative "objective" political truths. For example, if we reinterpret Rawls' standards in A Theory of Justice in this "truth-type talk", we can say that whether a normative claim is correct is matter of whether it is in accordance with principles acceptable to „reasonable”, "free”, and „equal” citizens in the „original position”; thus we can say that it is

3 There are also disagreements that have not been mentioned, the nature of which are more a matter of convention-for example, it seems that one would have to accept a majority verdict on whether to drive on the left-hand side of the road without further pondering the deep significance of this verdict. But, for example, the issue of euthanasia cannot be solved in the same manner: even if there were a very reliable procedure as to how to make correct decision on this issue, it would still make sense for somebody as a moral being to ponder the significance of its results. I owe this last point to David Estlund; see his "Beyond Fairness and Deliberation", p.187 in Deliberative Democracy ed. James Bohman and William Rehg, MIT Press, Cambridge, Massachusets 1997.

4 We can think of this as a settled paradigm of "normal science" in Kuhn's sense of this idea, given in his Scientific Revolutions. 
probably true that some instances of the minimal conception of common good, such as absence of war and famine, Pareto-efficient collective outcomes, the rule of law, economic prosperity, etc., would be chosen by individuals in a Rawlsiantype "original position", and this procedure would perhaps be the right way to attain them. In this sense the common good as described above would count as objective value. $^{5}$

Thus on the one side of the spectrum there are the truths of natural science, and on the other side there are putative truths of moral or political principles; I assume that truths along this spectrum could be correct answers to scientific and political questions; I also assume that correct answers are independent from the procedures we use to attain them. This ,standard-independency” attitude does not have to commit one to any sort of metatheoretical realism, whether scientific or moral. It is not that facts out there in the world make our judgments true or false; rather, practices themselves require certain proper standards on the basis of which we can attain successful predictions or correct answers in a particular domain. The proper function of these practices pertain to the nature of the field itself. Some practices, such as natural science inquiry, require a more exclusive group to be in charge, while some others, contrary to this, require a more inclusive group. The same applies to the institutional setup within which the groups work when they come to grips with an issue: when deciding on the acceptability of euthanasia, for example, the institutional setup should allow a wide democratic debate, whereas discussion, say, on the interpretation of experiments in physics on elementary particles does not. The question is, how should democratic society and the search for truth interlock along the abovementioned spectrum?

We shall see that, unlike in the case of one domain of scientific inquiry which we may, following Philip Kitcher, call „pure science”, 6 democratic procedure is directly relevant and perhaps crucial to solving practical collective problems in the right way as long as we as liberals believe that on average individuals are the best judges about what is good for them (this is both a moral and an epistemic assumption), and as long as we believe that the electorate as a whole - by using both deliberative and agregative methods - has a privileged status in solving collective practical problems.

An example can clarify this point: for epistemic reasons it should not be left to the expert(s) to decide for a society whether euthanasia should be legally allowed; on the other hand, suggestions as to how a bridge should be built, ceteris paribus, should rely more on estimates undertaken by capable engineers who could give figures offhand simply by relying on received knowledge from technical science and its constraints. This of course does not mean that authorities should not take into account anything else besides engineers' estimates (for example, the religious commitments of the population scattered around the construction site should come to the fore at some point); nor does it mean that the general public should not take into account experts' opinions on the possible

I follow here Helen Landemore's idea of political cognitivism and procedure-independent standards of correctness. See her book Democratic Reason (Chapter 8, Political Cognitivism: A Defense).

6 Philip Kitcher, Science, Truth and Democracy, Oxford University Press, Oxford, 2001, p. 87. 
effects of practicing euthanasia. The point is that experts should have the final say on how to build a bridge, whereas a democratic deliberation, followed by voting on referendum, could be necessary for eventually deciding the issue of euthanasia. And it should be no surprise that things should be done this way. The issue of euthanasia is morally heavily loaded and concerns every citizen in a political community; it requires maximal inclusiveness of the population and thus becomes a statutory or constitutional question.

Now, one might object to this that disagreements can occur even within a group of professional experts. One might wish to push further and claim that democratic deliberation, along with majority voting, is the best and most appropriate procedure in these cases and thus democracy stays with us even when dealing with „pure” science and experts working within its research projects. A sceptic might even push further and say that evidence for scientific claims is always insufficient, such that our decisions about what to believe are always crucially affected by our moral, social, political, and religious values. We can add to this problem a worry concerning the argument that science is never merely the pursuit of truth pure and simple, but rather the pursuit of those truths that scientists believe to be significant. ${ }^{7}$ One cannot isolate science from society, as some of the „scientific faithful” would have it, rather dogmatically.

But the question is whether conflicts about evidence in science require wide democratic public debate to be resolved. And the answer seems to be „no", because whether or not a wide electoral body finds them agreeable is not what matters. For example, the question of whether an elusive subatomic particle is detectable is not a moral issue, and thus it does not require public deliberation on the part of the entire electoral body. In addition, one more point needs to be added: from the assumption that ordinary citizens have equal right to exercise their freedom of ethical, religious, and political choice, one cannot conclude that everyone has an inherent ability to assess particular truth claims in science. When satisfying the virtue of truthfulness or accuracy in scientific endeavours (at least as far as "pure" science is concerned), it seems that moral, political, and religious judgments should be as far as possible kept away from any evaluation of evidence for conclusions.

\section{First Aspect: Scientific Truth and Democratic Society}

Generally, the very nature of scientific results about the natural world do not depend on whether we agree with them or not. They are not a matter of accidental circumstance or of shifting majority opinions of the general public. They are objective truths in the sense that one will always get the same result in the same experiment. For example, an experiment with gases shows that its result doesn't depend on who carries it out, but on independently existing physical laws relating to gases which say that pressure is a function of volume. Whether John or Peter, as experimenters, change the volume of the gas does not

7 For example, Kitcher (2001) argues that 'All kinds of considerations, including moral, social, and political ideals, figure in judgments about scientific significance' (p. 86). 
matter: if the temperature and amount of gas remain unchanged within a closed system, the pressure of a gas tends to decrease as the volume of a gas increases.

It also seems that our natural, common-sense epistemic attitude toward truth, scientific inquiry, and the objective world supports some form of scientific realism. Or, if we do not want to use the word 'realism', we can say that it in some sense supports some "correspondence" of the scientific theories. For example, when navigating, the fact that we end up so often where we want to go is best explained by the simple truth that our maps do represent the organisation of the land. Another examples abound: there are numerous successful scientific predictions. For example, planes stay up, a space module gets to the moon, e-mail goes through, etc. It is hard to conceptualize these successes unless we are in some sense right about the objective world around us. Thus in some sense, the "correspondence” aspect of scientific theories always stays with us even if we reject naive realism. The question is, what is left for democratic constituency to debate about science if we suppose that an exclusive professional group has the right to decide about what counts as objective knowledge?

What may concern democracy regarding objective scientific knowledge is transparency and the accessibility of accumulated knowledge when circumstances require that the public should be acquainted with its results. As Philip Kitcher puts it, when we say that scientific enquiry should be free, democracy may require that investigators be honest in the presentation of their findings, that conduct of experiments cannot override certain important moral values, etc. ${ }^{8}$ Let us turn now to some practical political problems.

\section{Second Aspect: Solving a Practical Social Problem-- Complexity, Uncertainty, Moral Sensitivity, and Fallibility}

Compared to the subjects matters of mathematics and natural science, social reality is a much messier domain. As Helene Landemore puts it: „When we discuss political opinions we are dealing with the domain where human beings deal with the risk and uncertainty of human life as a collective problem". 9 This is roughly equivalent to what Aristotle meant when he said in Nicomachean Ethics that when we are dealing with the domain of „human affairs”, we are dealing with things that could be different. ${ }^{10}$ In other words, the political domain is by definition an area that eludes apodictic judgements, where expertise is difficult to identify in advance. Going back to the previous example with John and Peter, we can see that in political matters things are not determined in the way they are determined in physics. Suppose it is true that progressive taxation increases social welfare among blue-collar workers. From this it does not follow that John and Peter, who are respected "authoritarian epistocrats" in country C, will necessarily manage to increase social welfare in $\mathbf{C}$ by implementing

$8 \quad$ See Kitcher (2001), p. 3.

9 See Lanemore (2013), p. 13.

10 Compare Aristotle's discussion of phronesis in Nicomachean Ethics 1140a24-1140b12. 
measures of progressive taxation. We wouldn't be very surprised if they failed as politicians or economists, so much we would have been surprised if they failed as experimenters in our previous example with gases in physics.

Politics is different from scientific inquiry because scientific inquiry requires conceptual and methodological rigor, with skills and knowledge usually acquired by special training in a system of very regulated institutions - such as schools, universities, research institutes, etc. On the other hand, politics is often about debate and disagreement on very delicate issues that determine us as moral beings, whether we are scientists, artists, or regular citizens. Thus political opinions often have an inter-subjective aspect that requires justification, debating, and public criticism. We discuss and try to change each others' minds about what we should value and what should be done on issues as diverse as abortion or waging war, because these issues are important to us as human beings. Thus nobody's judgement is taken for granted offhand, because each of us is prone to error due to the complexity of social reality as well as due to the fact that each of us is a protagonist in sensitive moral matters that involve value judgements.

\section{Deliberation as a Possible Way-Out}

Solving practical social problems in a democratic context means one more thing: decision reached by democratic process is only a tentative „truth", and is also subject to continuous revisions by present and future generations. This even applies to constitutional decisions. Although we intend to create the articles of a constitution so to last as long as possible, we cannot see their content as absolute truth. This should be obvious when we try to interpret the content of a constitution in order to decide what should be done regarding difficult issues, issues burdened by our deep value disagreements. Given the fact that democratic claims stops short of certainty, truth in democracy is not intended to silence dissenters on the grounds that they have mistaken opinions. The problem of the minority opinion can be accommodated by the fact that the democratic citizen, as a member of the majority, should be willing to listen to the other side, who hold opposing views and make concessions when "forced" to do so by better arguments coming from the minority. This practice can be developed along the lines of democratic discourse, the outline of which can be found in Habermas' famous idea of the "unforced force of a better argument". 11 Although this idea deserves wider attention as a separate issue, at this point we just want to say that the idea of deliberative democracy defended here excludes the possibility of silencing the minority by forcing it into accepting the majority view. In his work, Habermas develops a promising line on how the claims of normative rightness can be justified without relying on either the rigid notion of truth or on the idea of a winning majority in a democratic battle where rival parties wage a strategic political war. Assuming that the validity of a norm is justified only intersubjectively by exchanging arguments between individuals, Habermas

11 See his Moral Consciousness and Communicative Action, Cambridge, MIT Press, 1990 p. 160 as well as "Rightness versus Truth: On the Sense of Normative Validity in Moral Judgements and Norms" in Truth and Justification, Cambridge, MIT Press, 2003. 
establishes the standards of „communicative action”, 12 which excludes threats, promises, or any form of strategic action. What counts as a method of settling practical issues between truthful individuals belongs to mutual understanding and non-violent persuasion by better argument. The major difference between instrumental and communicative action is that the former is oriented towards success (whether in the natural or social world), whereas the latter is oriented toward reaching understanding in the social world. However, the idea of democratic discourse understood along the lines of communicative action does not have to give up on the idea of majority voting. Reaching a decision in politics can sometimes take a very long time, and if rational consensus stands beyond a community's reach, a majority voting procedure may be undertaken in order to avoid a political stalemate - provided that all available deliberative methods have been exhausted. ${ }^{13}$

\section{What structure does a group need to have in order to reach truth along the „spectrum of objectivity”?}

In the case of natural science we shall look to it that the group is composed of individuals whose level of reliability with respect to truth-tracking is relatively high (as was mentioned before, most of them should be properly trained, etc.). Obviously, this group will be less inclusive then the constituency of a polity. The group of experts in a field should have a coherent system of beliefs as far as possible and should update these beliefs according to the available evidence. On average, we would expect that the probability that this kind of group will reach the verdict that $\mathbf{p}$, when $\mathbf{p}$ is true, is very high, and much higher than random. Of course, this does not have to happen necessarily. The group of experts might collectively hold an inconsistent set of beliefs: there is no algorithm that guarantees the consistency of individually-held beliefs at the group level. By using the majority method of aggregation, even a group of experts can run into a set of incoherent statements about a certain topic in spite of the fact that each expert's individual set of judgements about the same topic was consistent. ${ }^{14}$

On the other hand, when it comes down to political issues such as capital punishment or euthanasia, we cannot just rely on experts but must include the general public for the following liberal reason: since it is a privilege held by a person to identify and pursue a conception of the good (most liberal thinkers hold this view. E.g., Mill, Kant, Rawls), democracy has to recognize the ability of each individual to live in accordance with her own judgement. Assuming that deliberation can reduce the risk of cycling majorities by making preferences on a collective level more single-peaked, ${ }^{15}$ assuming also that we can somehow

12 See The Theory of Communicative Action, Beacon Press, Boston, 1984.

13 Moral Consciousness and Communicative Action, p. 160.

14 See Pettit, P. and List, C., "Aggregating Sets of Judgments: Two Impossibility Results Compared", Synthese 140 (2004) 207-235.

15 See Fishkin, Christ, Luskin, McLean, "Deliberation, Single-peakedness, and the Possibility of Meaningful Democracy: Evidence from Deliberative Polls", LSE Online: October 2012, http:// eprints.lse.ac.uk/46863/ 
solve the problem of technicalities associated with deliberative undertaking on the large scale, we can expect a tendency towards correct collective decision by a democratic constituency. Democracy is understood here as a form of government the institutions of which are characterized by both deliberative procedure and majority rule; deliberative procedure is understood as a collective and mutual reason-giving activity between citizens who respect each other as free and equal, with the purpose of reaching a judgement based on reasons to which none could reasonably reject; where, when and how these two forms of collective decisionmaking are to be applied, depends on the context (context depends on the nature of the issue and on the character and level of the group. For example, policy advisers, jury in a higher court, nation-state constituency - these could all require different protocols when it comes to making collective decisions).

\section{3d Aspect: Creating Autonomous Citizens - the Relationship between the „Market-Place of Ideas” and Democratic Political Culture}

It is probably true that the majority of liberal thinkers view democracy as a social arrangement that favours autonomous citizens understood as critical individuals capable of sustaining and reinventing the democratic social context. This follows from the liberal and democratic ideal of public reason. The idea of public reason entails that the permissible use of state coercion requires public justification, which in turn means that coercion can be justified only if it is acceptable to free and equal citizens on the basis of reasons that they recognize as valid. The capacity to tell valid from invalid reasons pertains to a critical citizen prone to reflection about what is good for her as well as about the common good. ${ }^{16}$

From the institutional point of view, the problem of creating an autonomous citizen has much to do with how we understand and build the "market-place of ideas (Mill) in a society. More precisely, this task concerns the problem of what kind of „flow" of information matters in democracy. For example, is it that „more things being said" is necessarily better, or is it the quality of the information that matters? If quality matters, then what is important is not that everyone speaks, but that everything worth saying is be said. In this sense, ideally speaking, the market-place of ideas should approximate scientific inquiry and the structure of institutions associated with them. This structure, as Bernard Williams puts it, „involves increasingly high entry fees in terms of training, and also, necessarily, a powerful filter against the crank". ${ }^{17}$ But this is a very regulated market. It cannot be expected from public space.

Nevertheless, the institutional model of public fora in democratic society should move to a reasonable degree toward an environment that does not proliferate misinformation, trivialities, scandals, superstitions, or fancies, as well

16 As is well known, the idea of public reason can be found in John Rawls' work on political liberalism and public reason, as well as in the work of many other liberal authors working in this area (Joshua Cohen, for example).

17 Bernard Williams, Truth and Truthfulness, Princeton University Press, Princeton, p. 217. 
as other forms of behaviour that contribute to the failure of the proper working of the market-place of ideas - such as polarization, political silencing, etc. If one enters a proper democratic market-place of ideas, one should suppose that the public she addresses is capable of critical reflection. In other words, he or she is associated with people who are, to use Bernard Williams' expression, „ready not to be fooled", who do not believe everything they hear, and yet who may still retain great suspicion as to whether there is anything like objective, let alone absolute, truth in practical matters. ${ }^{18}$ In this sense one is creating herself as a democratic individual, capable of sustaining and recreating the democratic environment.

Thus public space should be arranged in such a way that when entering it one is at pains to convince the public into believing one's ideas. One's epistemic attitude is such that one's words and ideas are somewhat constrained so that they can be justified to all. When believing something, one assumes responsibility for responding to criticism, meeting challenges, and considering objections. In this sense, when entering democratic public space one is committed to truth implicitly. The justification of one's beliefs to the public counts as a part of the process of truth-acquisition. Obviously this environment requires conditions for deliberation process, such as mutual exchange of arguments, reciprocity, respects for interlocutors, etc. ${ }^{19}$ All this is the matter of political culture, the essential part of which is institutional setup and content. This brings us to the necessary institutional conditions for the proper working of the market-place of ideas, which is probably the most delicate issue for a democratic order. Let us now consider this problem.

\section{„Machiavellian” Features of a Government ${ }^{20}$}

Transparency is important in any democracy, and especially to deliberative democracy, where the virtue of truthfulness must come to the fore. However, as to the virtue of truthfulness, there is a principal discrepancy between the responsibility of a government on the one hand, and of private individuals on the other-there is no institutional algorithm that guarantees avoidance of this conflict. It is true that governments are responsible for the security of their citizens, and that this obligation cannot always be discharged without secrecy. Thus government may sometimes withhold information. But from this it does not follow that it may fool citizens around. The problem is how to control it.

$18 \quad$ Ibid, p. 1.

19 Again, Jurgen Habermas' project of "communicative action" and "discourse ethics" is of great help here. His outline of discourse ethics provides for a structure of argumentation that will yield rational and legitimate answers to moral questions without committing itself to substantive answers in this domain. When this form of argumentation is applied to the public domain of deliberative democratic politics, one can say, following Habermas, that laws are legitimate only if they are arrived at through discourses that are rational, which is to say characterized by inclusion, equality, and sincerity. See, for more on this, his Between Facts and Norms, MIT Press, 1996, pp. 107-110, where he discusses the process of validating legal norms.

20 The idea of calling this problem a "Machiavellian feature" of governmental workings was borrowed from Bernard Williams' paper "Truth, Politics and Self-Deception". See his In the Beginning was the Deed, Princeton University Press, Princeton, 2005, p. 157. 
One way to solve this problem is institutional, and is well known from the work of Montesquieu - this is the idea of a division of political power. Thus intelligence reports and security secrets that a democratic government acquires should be shared, perhaps by some members of a legislative part of the government, or even by opposition party members, or should be available through parliamentary hearings, etc. Perhaps such institutional practices may reduce the chance of misinformation, obscuring the truth, and so on. However, this is very difficult problem for any democracy. What makes it somewhat easier to accept these pessimistic conclusions is that, institutionally speaking, authoritarian regimes are less likely to successfully cope with this problem. The problem becomes even deeper when we think about the issue globally. For example, who is to scrutinize the work of a government's intelligence on an international level? Perhaps this can be done through a set of internationally-recognized institutions, or non-governmental organizations, or the free global press. The problem with this is that superpowers, even with a democratic institutional setup, dodge such issues and very often are not willing to comply to the standards of public reason in an international context. 